\title{
REACTION OF SPERMATOZOA WITH UTERINE AND SERUM GLOBULIN DETERMINED BY IMMUNOFLUORESCENCE
}

\author{
D. B. A. SYMONS \\ School of Agriculture, University of Cambridge
}

(Received 28th February 1967)

A naturally-occurring, heat-stable serum factor reacting with homologous and heterologous spermatozoa has been demonstrated by immunofluorescence (Beck, Edwards \& Young, 1962). The widespread incidence of the serum factor in both sexes of several species suggests that it may have some functional significance in sperm physiology. Spermatozoa do not normally contact blood serum but are suspended in fluids of the genital tract, which may contain protein of serum origin, for it has recently been shown that modified immune globulins pass into the rabbit genital tract (Kirton, Desjardins \& Hafs, 1966). The oestrous uterus exerts a modifying effect upon spermatozoa; the change, termed capacitation, being a necessary preparation of spermatozoa for fertilization (Austin, 1951; Chang, 1951). In the rabbit capacitation may involve a surface change of the sperm head (Bedford, 1965), although the character of the process is not yet clear. Immunofluorescence detects surface phenomena and has been used in the present study to examine the combination of rabbit globulin with uterine and Fallopian tube spermatozoa. Ejaculated rabbit spermatozoa and epididymal mouse and guinea-pig spermatozoa were also studied in vitro.

Oestrous rabbits of mixed breed were mated two or more times with two bucks of proven fertility. Seven to 12 hours p.c., the uterine and tubal contents were washed out separately with Ringer-Locke solution at $37^{\circ} \mathrm{C}$ and then recovered by centrifugation before resuspension in a small volume of RingerLocke. Suspensions were incubated with anti-rabbit globulin conjugated with fluorescein isothiocyanate (Difco) for $30 \mathrm{~min}$ at room temperature. After washing with two changes of Ringer's solution, spermatozoa were mounted in buffered glycerol, $\mathrm{pH} 7 \cdot 0$, for fluorescence microscopy (Reichert Fluorex; UG1/1.5 primary filter, Wratten 2B secondary filter, dark ground illumination).

Living ejaculated rabbit spermatozoa and epididymal mouse spermatozoa were incubated with heat-inactivated normal rabbit serum diluted with an

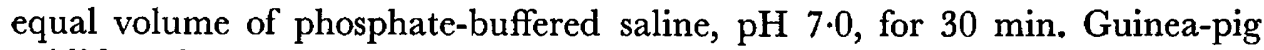
epididymal spermatozoa were similarly incubated with fresh and heat-inactivated homologous serum. After washing, spermatozoa were treated with fluorescein conjugate and examined as above. Control preparations of ejaculated 
rabbit spermatozoa and epididymal rabbit and mouse spermatozoa treated directly with conjugate were non-fluorescent, signifying that globulin was not absorbed by spermatozoa from the secretions of the male genital tract.

The results revealed a consistent pattern of fluorescence. In each sample of spermatozoa, whether recovered from the female tract or incubated with serum in vitro, a proportion remained non-fluorescent. These spermatozoa were invariably strongly motile. As degenerative changes in spermatozoa became apparent, typified by weak movements of the tail, enlargement and loosening of the acrosome, so fluorescence of the acrosome developed. The tail mainpiece of mouse spermatozoa incubated with rabbit serum was also fluorescent. Spermatozoa judged to be dead were the most strongly fluorescent. It, therefore, appeared that fluorescence of the sperm acrosome was a measure of the physiological decay of the cell. This hypothesis was tested by adding a suspension of actively motile spermatozoa to an excess of $95 \%$ ethanol immediately before incubation in serum and conjugate. Moderate to strong fluorescence of all the spermatozoa ensued. Clearly death of the cell, whether through ageing or induced by fixation, was conducive to its combination with serum globulin.

Spooner (1964) described the lysis of guinea-pig testis cells in autologous

TABLE 1

PERCENTAGE OF MOTILE AND NON-FLUORESCENT SPERM RECOVERED FROM UTERUS AND FALLOPIAN TUBE

\begin{tabular}{l|cc|ccc}
\hline & \multicolumn{2}{|c|}{$\%$ motile } & \multicolumn{2}{|c}{ \%on-fuorescent } \\
\hline $\begin{array}{l}\text { Fallopian tube spermatozoa } \\
\text { Uterine spermatozoa }\end{array}$ & 36 & $(70)$ & & 32 & $(62)$ \\
& 39 & $(253)$ & & 31 & $(97)$ \\
\hline
\end{tabular}

Figures in parentheses refer to total number of cells counted.

serum, the reaction being complement-dependent, and thus it was of interest to examine the effect of fresh guinea-pig serum in this system. Guinea-pig and rabbit spermatozoa were used. Fresh guinea-pig serum caused more rapid agglutination of spermatozoa than did heated serum, with some lysis and shedding of the acrosome. Aggregates of detached acrosomes were fluorescent, while spermatozoa that had shed the acrosome were non-fluorescent. A proportion of all spermatozoa treated with fresh guinea-pig serum remained actively motile and uncombined with serum globulin.

The reaction of naturally-occurring globulin with the acrosome therefore appears inversely related to the viability of the cell. The indications from these experiments are that physiological decay of spermatozoa precedes globulin absorption. However, the toxic effects of serum or spermatozoa are well known and it may be that combination with globulin is itself instrumental in the decay of the cell.

The fluorescence of uterine and tubal spermatozoa indicates that globulin is present in the female tract, which reacts similarly to that present in normal serum. In addition, it was observed that uterine spermatozoa which were fluorescent were those which were adherent to leucocytes or were being phago- 
cytosed. Non-fluorescent, motile spermatozoa remained free and showed little tendency to agglutinate, either to other spermatozoa or to leucocytes. Counts of motile and non-fluorescent spermatozoa in uterine and tubal washings are shown in Table 1. Within the limits imposed by the difficulty of counting motile cells and the delay introduced by treatment with conjugate, the figures for the two groups are positively related.

Phagocytosis by uterine leucocytes involved only decaying and dead spermatozoa, as judged by fluorescence. This implies that the reaction of spermatozoa with uterine fluid may be a prerequisite for their phagocytosis. Bedford (1965) has suggested that a change in the vital surface membrane of the sperm head occurring in the oestrous uterus might facilitate interaction between spermatozoa and leucocytes. The reaction between spermatozoa and globulin reported here may be the modifying effect which Bedford has proposed. It seems unlikely, however, that modification by globulin in the uterus is involved in capacitation. Firstly, spermatozoa which had reacted with globulin were ageing or dead and were being removed by phagocytosis, although it has been suggested that capacitation might include an additional selective process, for example disintegration of weak spermatozoa or phagocytosis of excessive spermatozoa (Adams \& Chang, 1962). Secondly, it is improbable that weakly motile spermatozoa would be capable of penetrating the cumulus cells investing the rabbit ovum. Spermatozoa able to penetrate the cumulus matrix are strongly motile (Austin, 1961). It seems likely, therefore, that uterine spermatozoa which remain free and impervious to globulin are those which are potentially capable of fertilizing the ovum.

I wish to thank Dr C. E. Adams for his criticism of the manuscript.

\section{REFERENCES}

Adams, C. E. \& Chang, M. C. (1962) Capacitation of rabbit spermatozoa in the fallopian tube and the uterus. F. exp. Zool. 151, 159.

Austin, C. R. (1951) Observations on the penetration of sperm into the mammalian egg. Aust. $\mathcal{F}$. scient. Res. B, 4, 581 .

Austin, G. R. (1961) Significance of sperm capacitation. Proc. IVth int. Congr. Anim. Reprod., The Hague, $4,723$.

BECK, J. S., Edwards, R. G. \& Young, M. R. (1962) Immune fluorescence technique and the isoantigenicity of mammalian spermatozoa. 7. Reprod. Fert. 4, 103.

BEDFord, J. M. (1965) Effect of environment on phagocytosis of rabbit spermatozoa. F. Reprod. Fert. 9, 249.

Chang, M. C. (1951) Fertilizing capacity of spermatozoa deposited into the fallopian tubes. Nature, Lond. 168, 697.

Kirton, K. T., Desjardins, C. \& Hafs, H. D. (1966) Transfer of antibody into rabbit seminal plasma and uterine fluid. F. Anim. Sci. 25, 926.

Spooner, R. L. (1964) Cytolytic activity of the serum of normal male guinea pigs against their own testicular cells. Nature, Lond. 202, 915. 